\title{
The Use of Intravenous Prostaglandins in Extremity Ischemia
}

\author{
Vimalin Samuel ${ }^{1} \quad$ Dheepak Selvaraj $^{1} \quad$ Albert Abhinay Kota $^{1}$ Edwin Stephen ${ }^{2}$ \\ 1Department of Vascular Surgery, Christian Medical College,
Vellore, Tamil Nadu, India
2Department of Vascular Surgery, Sultan Qaboos University,
Muscat, Oman \\ Address for correspondence Dheepak Selvaraj, MCh, Department \\ of Vascular Surgery, Christian Medical College, Vellore, Tamil Nadu \\ 632004, India (e-mail: vascular@cmcellore.ac.in).
}

Int J Recent Surg Med Sci 2019;5:53-57

\begin{abstract}
Introduction Prostaglandins are potent vasoactive agents with other properties of vasodilatation, fibrinolysis and inhibition of platelet aggregation. Prostaglandin E1 (PGE1) has been used in the treatment of peripheral vascular diseases (PVD), mainly in patients with nonreconstructable PVD and also as an adjunct when there is residual ischemia after revascularization. Our experience in using PGE1 at a tertiary care center in South India is presented here along with a review of literature.

Materials and Methods Patients presenting to us with nonreconstructable critical limb ischemia underwent prostaglandin infusion after meeting selection criteria.

Results Of the 142 patients enrolled into the study, 48 completed the study. There was a shift in the profile of patients from critical limb ischemia to claudicants or minor tissue loss.

Keywords

Discussion In the absence of options to treat patients with nonreconstructable criti-

- alprostadil cal limb ischemia, prostaglandin infusion offers a viable option for treatment.

- ischemia Conclusion PGE1 infusion is an option for patients with nonreconstructable critical - PGE1 limb ischemia.
\end{abstract}

\section{Introduction}

Prostaglandins (PGs) were discovered in 1935 by Von Euler as a blood pressure-lowering substance from the prostate gland secretion. ${ }^{1}$ PGs are potent vasoactive agents with actions that depend on the species, organ tested, and the PG used. They are synthesized from 20-carbon polyunsaturated fatty acids containing three, four, or five double bonds. These fatty acids are present in the phospholipids of the cell membranes of all mammalian tissues. ${ }^{2}$ Prostaglandin E1 (PGE1) is a major PG found in human semen that functions as a vasodilator, inhibitor of platelet aggregation, and a fibrinolytic agent. The compound also inhibits monocyte and neutrophil function, suggesting that PGE1 will also have anti-inflammatory effects. Recent research has detected additional actions of PGE1 and prostacyclin analogs which might be relevant to its clinical efficacy. This includes inhibition of expression of adhesion molecules (E-selectin, ICAM-1, and VCAM-1), release of inflammatory cytokines (TNFalpha, MCP-1), matrix components, and generation and release of growth factors (CYR61, CTGF). These

DOI https://doi.org/

10.1055/s-0039-3402525

ISSN 2455-7420. actions may also contribute to the long-term effects of PGE1, particularly in more advanced stages of PAD. Gene-expression experiments with chemically stable prostacyclins and PGE1 suggest that several genes in vascular smooth muscle cells and fibroblasts are modified by PGs at the transcriptional level. ${ }^{3}$ PGE1 has been reported to benefit patients with significant peripheral vascular disease and limb-threatening ischemia. ${ }^{4}$ Both intravenous (IV) or intra-arterial (IA) routes have been described. Previously, the use of an implantable port with its catheter placed IA for PGE1 infusion has been described. ${ }^{5}$ The disadvantages of the IA infusion are local side effects as rubor, swelling, and pain; however, the IV route needs a significantly increased PGE1 dosage (up to four times) to achieve the same therapeutic dose levels as the arterial route. A total of $90 \%$ of PGE1 undergoes first pass metabolic degradation by the lung parenchyma. The increased dosage makes the IV treatment especially in patients with borderline cardiac or renal function, problematic. With IA application of $20 \mu$ of PGE1 (a quarter of the dose required for IV delivery), systemic adverse effects, such as hypotension (due to vasodilatation), lung edema, or

(C2019 Medical and Surgical

License terms

Update Society 
cardiac failure, are significantly decreased. The therapeutic effects of the substance, therefore, are probably more due to other mechanisms of action than due to hemodynamic effects. ${ }^{6}$

The benefits of prostanoids in claudicants due to peripheral arterial disease are doubtful. The largest available systematic review concluded that the quality of studies and the overall evidence available are insufficient to determine whether or not patients with intermittent claudication derive clinically meaningful benefit from the administration of prostanoids. Further well-conducted randomized, double blinded trials with a sufficient number of participants to provide statistical power are required to answer this question. ${ }^{7}$

Critical limb ischemia is a late stage of peripheral arterial disease characterized by pain at rest and tissue loss. Treatment of critical leg ischemia consists primarily of revascularization by angioplasty or reconstructive surgery. There are groups of patients in whom, on account of poor distal reformation, both may not be an option. This picture is mostly seen in patients with Thromboangiitis obliterans. The ICAI study group, following a randomized, controlled, open label trial with PGE1, suggested that short-term treatment with Alprostadil (PGE1) provides patients with critical leg ischemia clinical benefit that is apparent in the short term but decreases over time. ${ }^{8}$

A systematic review of the numerous heterogeneous studies published in 2018 concluded that prostanoids have no effect on the incidence of total amputations when compared against placebo. There was moderate-quality evidence that showed small beneficial effects of prostanoids for restpain relief and ulcer healing when compared with placebo. Additionally, moderate-quality evidence showed a greater incidence of adverse effects with the use of prostanoids and low-quality evidence suggests that prostanoids have no effect on cardiovascular mortality when compared with placebo. None of the included studies reported quality of life measurements. The balance between benefits and harms associated with the use of prostanoids in patients with critical limb ischemia with no chance of reconstructive intervention is uncertain; therefore careful assessment of therapeutic alternatives should be considered. ${ }^{9}$

\section{Materials and Methods}

PGE1 has been used in our institution since 2010 to relieve the rest pain, heal ulcers in ischemic legs. It was also used for limb salvage in acute on chronic ischemia patients with viable limbs but not suitable for reconstruction surgery and interventions or those that had failed reconstructive procedures. Patients from the year 2011 to 2016 were enrolled into this nonrandomized observational study. The inclusion criteria were patients with nonreconstructable peripheral arterial disease and critical limb ischemia and/or those that had undergone reconstructive procedures but still had persistent pain, as deemed by four vascular consultants. Patients with reconstructable disease, acute or chronic infection, significant ischemic heart disease with ejection fraction less than $40 \%$ or cardiac rhythm disorders, tachycardia due to PGE1 infusion, were excluded. All infusions were done in the vascular ward. Injection Alprostadil (PGE1) 500 mcg was administered in three divided doses, each IV infusion over 6 hours. The study patients were subjected to one dose per day during which pulse rate and blood pressure were monitored by a bedside monitor. The study required the patient to be admitted in the ward for 3 days. The patient was required to complete six doses of PGE1 infusion, one every month for 6 months. The patients were graded with the Rutherford grading of chronic limb ischemia preinfusion at the end of the study. ${ }^{10}$ Preinfusion and postinfusion ankle brachial pressure index (ABPI) were also done to provide objective measure of improvement. Amputations were defined as major-any amputation at and above the metatarsal level and minor amputations as any amputation done below the level of the metatarsals.

\section{Results}

There were a total of 142 patients enrolled into the study over the course of 6 years ( - Fig. 1). However, due to demographic reasons, 94 patients were lost to follow-up or did not complete the required six doses of PGE1 infusion. Hence the final analysis was only done on 48 patients that completed the study.

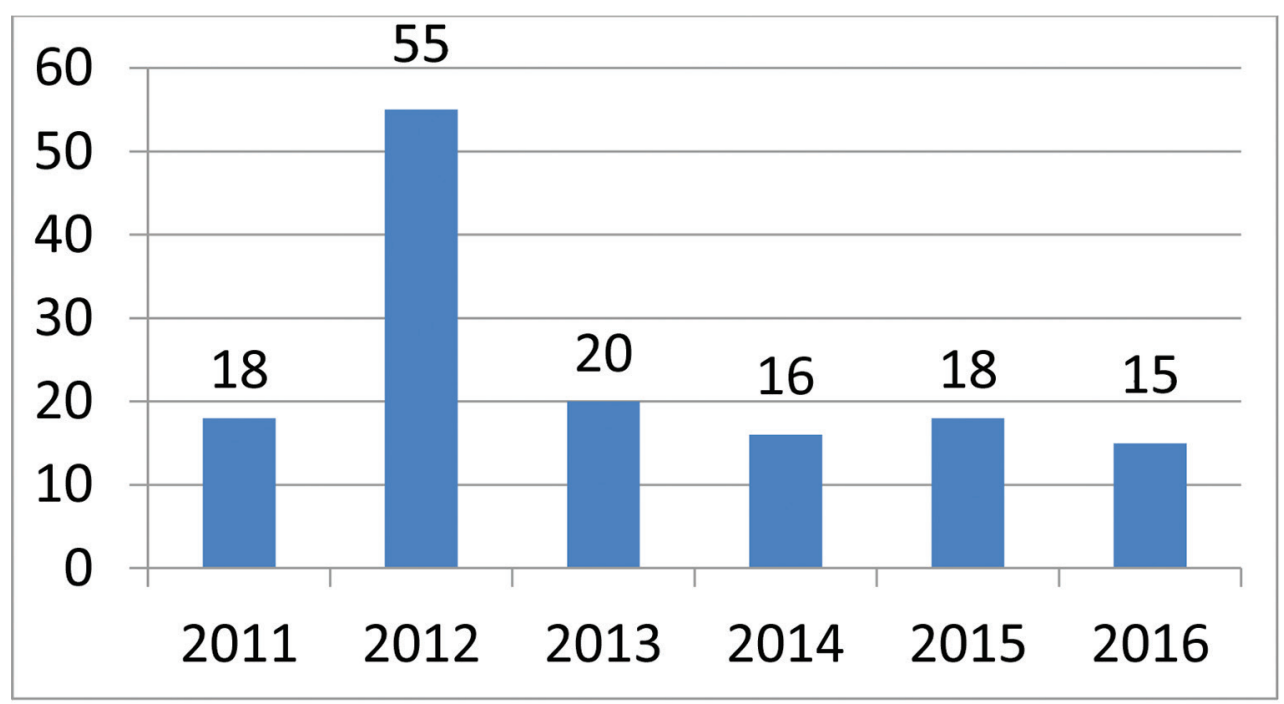

Fig. 1 Number of patients enrolled during the duration of the study. 
The patient profile in our study group is shown in - Table 1. The study group $(n=48)$ was divided into grades of ischemia based on the Rutherford Grading of Chronic Limb Ischemia ${ }^{10}$ preinfusion and after completion of six doses of PGE1 (-Figs. 2-4). There was a shift in the number of patients with higher grades of ischemia to lower grades of

Table 1 Patient profile

\begin{tabular}{|l|l|}
\hline \multicolumn{2}{|c|}{ Patient profile, $n=48$} \\
\hline Age distribution (y) & Number \\
\hline $20-40$ & 18 \\
\hline $40-60$ & 22 \\
\hline$>60$ & 8 \\
\hline Extremity involvement & 2 \\
\hline Upper limb & 46 \\
\hline Lower limb & \multicolumn{2}{|l|}{} \\
\hline Segment involved & 31 \\
\hline Infrapopliteal & 17 \\
\hline Femoropopliteal & 0 \\
\hline Aortoiliac & \multicolumn{2}{|l|}{} \\
\hline Diagnosis at presentation & 31 \\
\hline TAO & 16 \\
\hline Atherosclerotic PAD & 1 \\
\hline Others & 12 \\
\hline Comorbid illnesses & 1 \\
\hline DM/HTN & 34 \\
\hline IHD & \multicolumn{2}{|l|}{} \\
\hline Others & 12 \\
\hline None & \\
\hline
\end{tabular}

Abbreviations: DM, diabetes mellitus; HTN, hypertension; IHD, ischemic heart disease; PAD, peripheral arterial disease; TAO, thromboangiitis obliterans. ischemia. There were a few patients that underwent amputations (both major and minor); however, a majority of them were either minor or no amputations at all. The average rise in ABPI at the end of the study was 0.2 in patients involving either the infrapopliteal and femoropopliteal segments (-Fig. 5). Five patients underwent an adjunct sympathectomy. Two patients underwent an attempt at angioplasty and had persistent pain postprocedure. There were no PGE1 infusion-related complications in any of the patients.

\section{Discussion}

An ideal approach to the treatment of critical limb ischemia is the elimination or bypass of the occlusions/stenosis, but there may be situations in which it is often impossible or else, fails. A good alternative in these cases is to attempt to change the consequences of low perfusion pressure on the distal microcirculation sufficiently by pharmacotherapy to relieve rest pain and avoid amputations. In fact, the primary goal of therapy is to provide the patient with a viable limb. The prostanoid investigated in the largest number of patients with advanced chronic limb ischemia in controlled randomized studies is Iloprost. ${ }^{11}$ The studies that have looked at PGE1 infusion are heterogeneous and enough evidence to make a significant change in practice is lacking. The number of patients that were enrolled into the study gradually decreased over a period of 6 years as our endovascular armamentarium increased, thus making patients that were previously considered nonreconstructable, reconstructable. Our study showed a significant shift in the grade of ischemia toward lower grades, thus making patients with critical limb ischemia into claudicants. This is associated with decreased number of amputations, which is the primary goal of treatment of peripheral arterial disease. At our institution, the standard protocol for the management of patients with claudication is best medical therapy which does not include PGE1

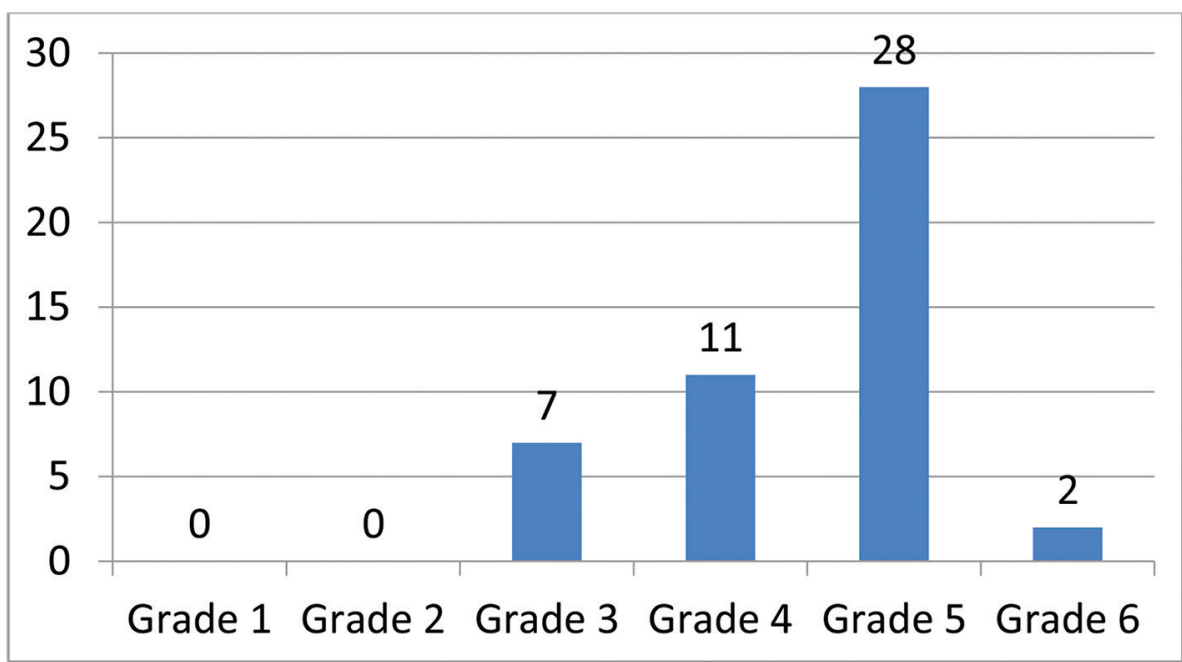

Fig. 2 Pre-PGE1 distribution of patients according to Rutherford Grade 1-6. PGE1, prostaglandin E1. 


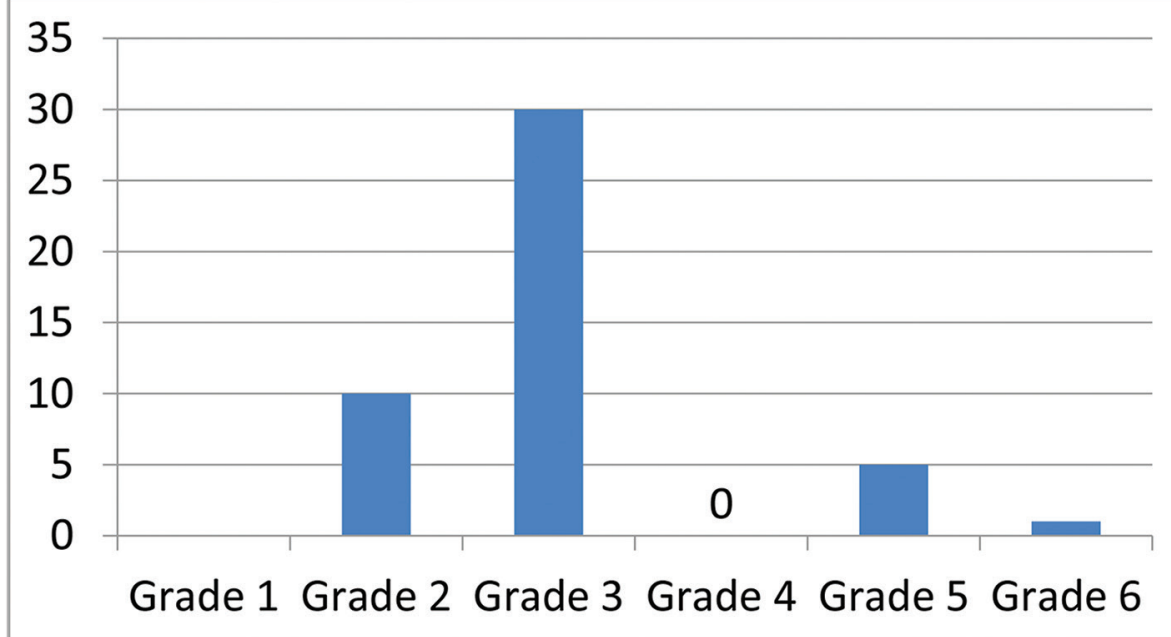

Fig. 3 Post-PGE1 distribution of patients according to Rutherford Grade 1-6. PGE1, prostaglandin E1.

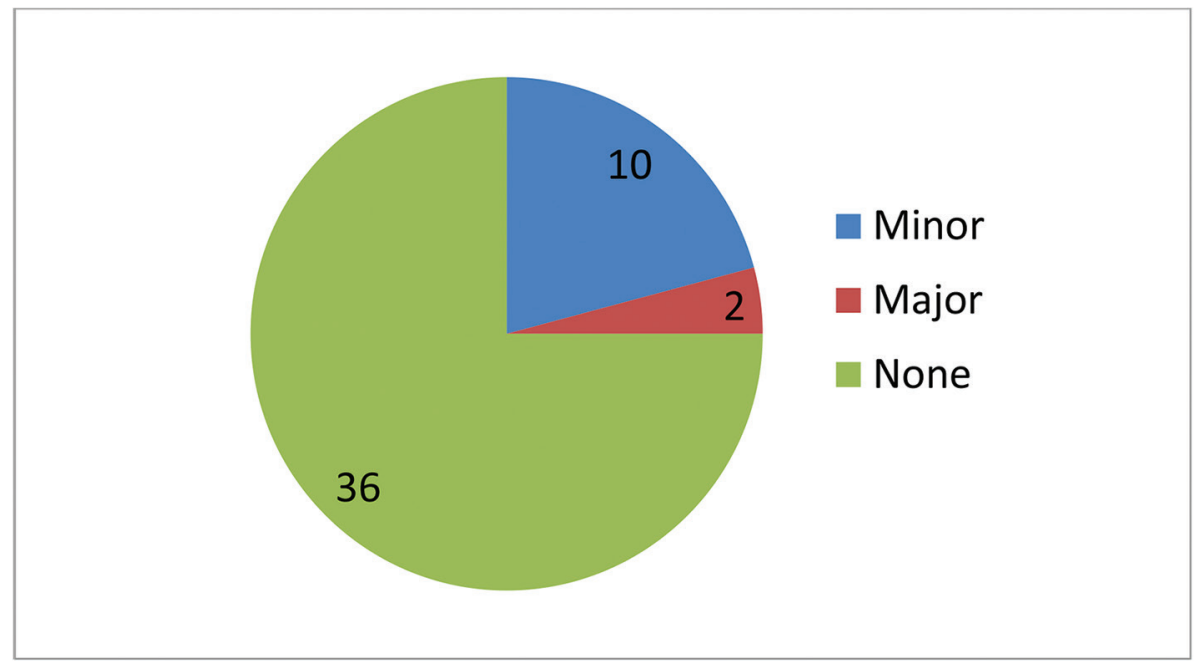

Fig. 4 Number of patients who underwent amputation (major and minor).

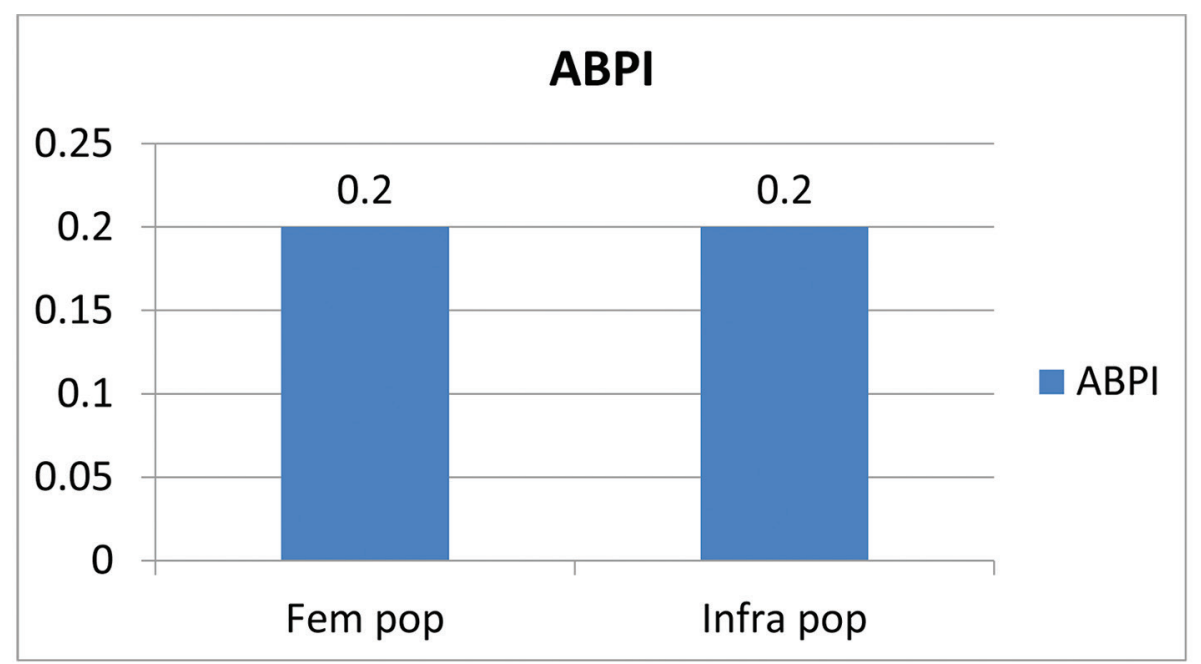

Fig. 5 Average rise in $\mathrm{ABPI}$ at the end of the study. $\mathrm{ABPI}$, ankle brachial pressure index. 
infusion, unless the claudication is disabling. Hence there were only a few claudicants entered into the study. The rise in ABPI is difficult to interpret but it is safe to assume that there was significant improvement in perfusion pressure. Whether that translates into prolonged amputation free survival can be seen only with longer follow-up. The role of transcutaneous partial pressure of oxygen (TCPO2) in assessing perfusion pressure needs to be studied. But it is safe to assume that there is a definite short-term benefit with the use of PGE1. Although these results may look impressive, it is also important to be aware of their limitations. Total ulcer healing was not used because it was too uncommon an event after a few weeks of treatment. The other limitation of the study is the number of study drop outs. Our practice is to administer the first dose of PGE1 in our hospital, request the patients to procure four doses at discharge, to continue the infusion at the nearby medical center, once a month, with a written protocol. The patient is encouraged to return to our center for the sixth and final dose. However, our study patients were from a wide range of demographic distribution, making follow-up and return for the sixth dose, difficult. Further, PGE1 is not an easily available drug and is expensive, leading to a high dropout rate. The role of PGE1 in patients with TAO has not been studied earlier and these results may suggest that there is benefit to a select group. There is no available technique for identifying those who will respond, but its relative safety suggests that it should be tried in all such patients unless an early amputation is clearly unavoidable.

\section{Conclusion}

PGE1 infusion provides an acceptable therapeutic alternative to patient with nonreconstructable critical limb ischemia with minimal complications.

\section{Conflict of Interest}

None declared.

\section{References}

1 USV Euler. An adrenaline-like action in extracts from the prostatic and related glands. J Physiol 1934;81(1):102-112

2 Moncada S, Vane JR. Arachidonic acid metabolites and the interactions between platelets and blood-vessel walls. N Engl J Med 1979;300(20):1142-1147

3 Schrör K, Hohlfeld T. Mechanisms of anti-ischemic action of prostaglandin E1 in peripheral arterial occlusive disease. Vasa 2004;33(3):119-124

4 Balzer K, Rogatti W, Rüttgerodt K. Efficacy and tolerability of intra-arterial and intravenous prostaglandin E1 infusions in occlusive arterial disease stage III/IV. Vasa Suppl 1989;28:31-38

5 Strecker EP, Boos IB, Ostheim-Dzerowycz W, Heber R, Vetter SC. Percutaneously implantable catheter-port system: preliminary technical results. Radiology 1997;202(2):574-577

6 Brecht T, Ayaz M. Circulation parameters during intravenous and intra-arterial administration of increasing doses of prostaglandin E1 in healthy subjects. Klin Wochenschr 1985;63(23):1201-1204

7 Robertson L, Andras A. Prostanoids for intermittent claudication. Cochrane Database Syst Rev 2013;( 4):CD000986

8 Prostanoids for chronic critical leg ischemia. A randomized, controlled, open-label trial with prostaglandin E1. The ICAI Study Group. Ischemia Cronica degli Arti Inferiori. Ann Intern Med 1999;130(5):412-421

9 Vietto V, Franco JV, Saenz V, Cytryn D, Chas J, Ciapponi A. Prostanoids for critical limb ischemia. Cochrane Database Syst Rev 2018;1:CD006544

10 Rutherford RB, Baker JD, Ernst C, et al. Recommended standards for reports dealing with lower extremity ischemia: revised version. J Vasc Surg 1997;26(3):517-538

11 ScienceDirect. Pharmacotherapy for critical limb ischemia. Available at: https://www.sciencedirect.com/science/article/ pii/S1078588400800337. Accessed October 14, 2019 